\title{
A fish eye view of the mirror test
}

\section{Jennifer Vonk ${ }^{1}$}

Published online: 17 June 2019

(C) The Psychonomic Society, Inc. 2019

\section{Summary}

Reactions to a recent study suggesting that cleaner wrasse can pass the mirror self-recognition test (Kohda et al. in PLOS Biology, 17(2), e3000021, 2019) reveal more about scientists' biases than about self-awareness. Scientists should base conclusions about species' abilities based on the corpus of data on that species rather than on a single test or preconceived expectations based on phylogeny alone.

Keywords Fish $\cdot$ Mirror self recognition $\cdot$ Speciesism

Issues with the mirror-self-recognition (MSR) test of selfawareness can be traced back at least two decades (e.g., De Veer \& Van den Bos, 1999). Animals must progress to a stage where they show self-directed behaviors in the presence of the mirror prior to being surreptitiously marked. Attention to the mark then validates the self-exploration shown in the previous stage. However, it is challenging to use this method in fish, which cannot easily examine themselves. Interestingly, a study purporting to show MSR in the cleaner wrasse has reawakened the debate over whether the paradigm is appropriate for testing self-awareness, which was long considered a hallmark of uniquely human cognition (Kohda et al., 2019). The wrasse showed the typical social behavior progressing to idiosyncratic behaviors in front of the mirror. After being marked with dye, they attempted to scrape off colored but not transparent marks only in the presence of the mirror. Reaction to the study says as much about scientists' biases, such as speciesism, as it does about the validity of the paradigm itself. The problem is not that scientists are skeptical when distantly related species pass tests of abilities hitherto attributed only to humans; but rather that scientists may not be critical enough when evidence for such abilities is championed in our closest kin. As Kohda et al. (2019) argue, a critical interpretation of results from MSR tests is useful only if it is applied to all species tested (including human children), and not only when affirmative results come from insects, birds, and, now, fish.

Jennifer Vonk

vonk@oakland.edu

1 Psychology, Oakland University, Rochester, MI, USA
Perhaps the important lesson is that scientists should be cautious when attributing "intelligence" to various behaviors. Does the kind of body self-awareness demonstrated in the mark test really equate to concepts about one's self as a psychological entity? De Waal (2019) conflates these distinct aspects of self-awareness in his commentary despite making the insightful observation that we should not expect all-or-none cognition, but should look for components of capacities in other species. De Waal (2019) and others raised concerns with the study itself, including the use of an irritating marker and the likelihood that cleaner wrasse view the mark as a parasite. But these concerns with whether the fish even pass the test are secondary to the issue of what we should conclude about their cognition if we consider them to have passed.

The authors themselves question whether their results indicate that cleaner wrasse are self-aware (Kohda et al., 2019). This well founded concern is objectionable if it is considered more legitimate based on unvalidated assumptions about which species might be capable of specific cognitive feats. Appropriate justification could be garnered through consideration of other known aspects of a species' cognition or morphology. For example, if an organism cannot accurately predict the behaviors of conspecifics based on patterns of observable behavior, it is unlikely that they are attributing reputations or mental states to these conspecifics. Interestingly, some corvids - one of the few taxonomic groups attributed the kind of "advanced cognition" typically reserved for primates (e.g., Taylor, 2014) - have been granted self-awareness using the MSR test (e.g., Medina, Taylor, Hunt, \& Gray, 2011), but scientists have likely been willing to do so because of the corpus of data suggesting sophisticated cognitive abilities (Taylor, 2014). Considering the larger body of work on a 
species is recommended, so long as abilities in other species are not dismissed simply because a larger body of evidence does not yet exist. Thus, it is defensible to form different expectations for different species, but not to waive or dismiss a criterion because of those expectations.

It can be justified to examine species that would not be expected to exhibit the given capacity as a control test case of sorts. For example, it is crucial to test relatively asocial species on tests of social cognition because in the absence of such tests we cannot conclude that social species have superior social cognitive abilities. If species fail the tests they are expected to fail, it adds validity to the measurement. But, in the case of fish meeting the criterion for self-awareness, we can either reject the test and acknowledge that we have been too lenient in our criteria for other species, or we can reassess our assumptions about the tested species, as acknowledged by the authors.

The question of whether legitimate affirmative results speak to "intelligence" is critical to the future of the field of animal cognition. Scoring various species in terms of 'intelligence' is not useful for understanding the evolution of diverse cognitive traits that vary in adaptive value depending on morphology, environmental niches, and social structures. A fish that can act to disable a parasite is exhibiting adaptive behavior. A fish that can recognize itself in a mirror, or exhibit a selfconcept, likely is not. Comparative cognition would gain more from a better understanding of which selective pressures give rise to behaviors and cognition than it would by an almost single-minded focus on finding evidence for potentially uniquely human capacities. Identifying components of traits that reveal how and when abilities emerged across evolutionary history will ultimately tell us more about the evolution of human cognition than the search for analogous traits in species that have faced profoundly different selective pressures.

Scientists should think carefully about which species are selected for testing particular abilities with a clear rationale as to why the ability may have arisen in this species rather than simply trying to find a shocking or exciting result. Is it sensible to test species that cannot manipulate their own bodies with the MSR paradigm? Compelling evidence comes from studies where subjects examine parts of their bodies they otherwise could not see, such as teeth. Cetaceans and fish can open their mouths in front of the mirror, but are they able to see inside their mouths as primates can? Perhaps aquatic animals swim past mirrors exposing the marked area of the body because doing so results in a more interesting visual experience, not because they understand that their movements are causing the changing image in the mirror or that the image reflects themselves.

Perhaps the most useful outcome of this study will be if it compels scientists to seek more species-appropriate methodologies for extending the exploration of well-studied topics to understudied species. If a cleaner wrasse passing the criteria for self-recognition in the MSR paradigm is unsettling, then we need to develop a test suitable for fish. Our goal should be to apply the same standards for what constitutes evidence of a capacity across species, not to "raise the goalposts" when evidence for previously deemed sophisticated cognitive abilities is reported in surprising species, such as fish. In the end, these controversies are helpful if they cause us to question our motives and criteria.

Although the reactions to Kohda and colleagues' findings may reveal biases on the part of scientists, it is appropriate to cast aside a paradigm that fails to differentiate the behavior of animals that show other indications of self-awareness from those that do not. Work with human children is valuable because we know that they begin to refer to themselves using personal pronouns at around the same age at which they recognize themselves in the mirror. An immense body of developmental work reveals patterns in children's performance across various cognitive tests and abilities, which is lacking in other species. As with human studies, tests should be validated against other measures assumed to tap into the same capacities. If species pass only one test of a construct but fail many others, the test likely does not provide a good measure of that capacity. Thus, intraspecies comparisons are at least as important as interspecies comparisons. We must compile many studies of many different capacities to determine which aspects of cognition are associated and what factors predict their presence rather than assuming that a single set of data can reveal a clear window into other minds.

\section{References}

De Veer, M. W., \& Van den Bos, R. (1999). A critical review of methodology and interpretation of mirror self-recognition research in nonhuman primates. Animal Behaviour, 58(3), 459-468. https://doi.org/ 10.1006/anbe.1999.1166

de Waal, F. B. M. (2019). Fish, mirrors, and a gradualist perspective on self-awareness. PLOS Biology, 17(2), e3000112. https://doi.org/10. 1371/journal.pbio.3000112

Kohda, M., Hotta, T., Takeyama, T., Awata, S., Tanaka, H., Asai, J., \& Jordan, A. L. (2019) If a fish can pass the mark test, what are the implications for consciousness and self-awareness testing in animals? PLOS Biology, 17(2), e3000021. https://doi.org/10.1371/ journal.pbio.3000021

Medina, F. S., Taylor, A. H., Hunt, G. R., \& Gray, R. D. (2011). New Caledonian crows' responses to mirrors. Animal Behaviour, 82(5), 981-993. https://doi.org/10.1016/j.anbehav.2011.07.033

Taylor, A. H. (2014). Corvid cognition. WIREs Cognitive Science, 5(3), 361-372. https://doi.org/10.1002/wcs.1286

Publisher's note Springer Nature remains neutral with regard to jurisdictional claims in published maps and institutional affiliations. 